\title{
Radio properties of the low surface brightness SNR G65.2+5.7
}

\author{
L. Xiao ${ }^{1}$, W. Reich ${ }^{2}$, E. Fürst ${ }^{2}$, and J. L. $\operatorname{Han}^{1}$ \\ ${ }^{1}$ National Astronomical Observatories, Chinese Academy of Sciences, Jia-20, Datun Road, Chaoyang District, Beijing 100012, \\ PR China \\ e-mail: xl,hjl@bao.ac.cn \\ 2 Max-Planck-Institut für Radioastronomie, Auf dem Hügel 69, 53121 Bonn, Germany \\ e-mail: efuerst, wreich@mpifr-bonn.mpg.de
}

Received 23 January 2009 / Accepted 9 April 2009

ABSTRACT

Context. SNR G65.2+5.7 is one of few supernova remnants (SNRs) that have been optically detected. It is exceptionally bright in $\mathrm{X}$-rays and the optical [O III]-line. Its low surface brightness and large diameter ensure that radio observations of SNR G65.2+5.7 are technically difficult and thus have hardly been completed.

Aims. Many physical properties of this SNR, such as spectrum and polarization, can only be investigated by radio observations. Methods. The $\lambda 11 \mathrm{~cm}$ and $\lambda 6 \mathrm{~cm}$ continuum and polarization observations of SNR G65.2+5.7 were completed with the Effelsberg $100-\mathrm{m}$ and the Urumqi $25-\mathrm{m}$ telescopes, respectively, to investigate the integrated spectrum, the spectral index distribution, and the magnetic field properties. Archival $\lambda 21 \mathrm{~cm}$ data of the Effelsberg 100-m telescope were also used.

Results. The integrated flux densities of G65.2+5.7 at $\lambda 11 \mathrm{~cm}$ and $\lambda 6 \mathrm{~cm}$ are $21.9 \pm 3.1 \mathrm{Jy}$ and $16.8 \pm 1.8 \mathrm{Jy}$, respectively. The power-law spectrum $\left(S \sim v^{\alpha}\right)$ is well fitted by $\alpha=-0.58 \pm 0.07$ from $83 \mathrm{MHz}$ to $4.8 \mathrm{GHz}$. Spatial spectral variations are small. Along the northern shell, strong depolarizion is observed at both wavelengths. The southern filamentary shell of SNR G65.2+5.7 is polarized by as much as $54 \%$ at $\lambda 6 \mathrm{~cm}$. There is significant depolarization at $\lambda 11 \mathrm{~cm}$ and confusion with diffuse polarized Galactic emission. Using equipartition principle, we estimated the magnetic field strength for the southern filamentary shell to be between $20 \mu \mathrm{G}$ (filling factor 1) and $50 \mu \mathrm{G}$ (filling factor 0.1). A faint HI shell may be associated with the SNR.

Conclusions. Despite its unusually strong X-ray and optical emission and its very low surface brightness, the radio properties of SNR G65.2+5.7 are found to be typical of evolved shell-type SNRs. SNR G65.2+5.7 may be expanding in a pre-blown cavity as indicated by a deficit of $\mathrm{HI}$ gas and a possible HI-shell.

Key words. ISM: supernova remnants - ISM: magnetic fields - radio continuum: ISM - radiation mechanisms: non-thermal

\section{Introduction}

SNRs of low radio surface brightness and large diameter are difficult to identify in radio continuum surveys. The weakest source detected in our Galaxy is SNR G156.2+5.7 (Reich et al. 1992), which has a surface brightness $\Sigma_{1 \mathrm{GHz}} \sim 5.8 \times$ $10^{-23} \mathrm{~W} \mathrm{~m}^{-2} \mathrm{~Hz}^{-1} \mathrm{sr}^{-1}$, and SNR G65.2+5.7 is a similar weak object, whose low surface brightness may be caused by a low ambient magnetic field strength or a low electron density. In most cases, the supernova has exploded in a low-density medium, for instance in an interarm region or in a pre-existing cavity. In the case of a pre-existing cavity substantial radio emission is visible for only a relatively short time interval as the shock collides with the dense shell.

Gull et al. (1977) first identified G65.2+5.7 as a large diameter SNR located in the Cygnus region in an optical emission line survey, where it appeared to be exceptionally bright in [O III]. This is indicative of high shock velocities typically for an expanding SNR shell. Reich et al. (1979) used the Effelsberg $100-\mathrm{m}$ telescope at $\lambda 21 \mathrm{~cm}$ to map G65.2+5.7 and confirmed its identification as a SNR by its non-thermal emission.

\footnotetext{
* Figures 1 and 2 (6 and $11 \mathrm{~cm}$ maps) are available in FITS format at the CDS via anonymous ftp to cdsarc.u-strasbg.fr (130.79.128.5) or via
}

http://cdsweb.u-strasbg.fr/cgi-bin/qcat?J/A+A/503/827
G65.2+5.7 is a bright soft X-ray source for which Shelton et al. (2004) analysed ROSAT PSPC data, classifying the SNR as a "thermal composite" object with a cool, dense shell without X-ray emission. Bright centrally peaked thermal X-ray emission dominates the interior of these SNRs, although they are rare. W44 is another example (Cox et al. 1999), which is younger than G65.2+5.7.

Distance estimates were made by various authors. Boumis et al. (2004) used proper motion and expansion measurements of the remnant's optical filament edges and obtained $770 \pm 200 \mathrm{pc}$. In the following, we assume a distance of $800 \mathrm{pc}$. The apparent size of G65.2+5.7 is about $4^{\circ} \times 3.3$ (Gull et al. 1977), corresponding to a physical size of $56 \mathrm{pc} \times 46 \mathrm{pc}$. The centre of the SNR is located about $80 \mathrm{pc}$ above the Galactic plane. G65.2+5.7 appears to be an evolved object, although its age is uncertain: It may be as old as $3 \times 10^{5}$ years if one assumes an average shock velocity of $\sim 50 \mathrm{~km} \mathrm{~s}^{-1}$ (Gull et al. 1977; Reich et al. 1979). Optical observations show the velocities in the range between $90 \mathrm{~km} \mathrm{~s}^{-1}$ and $140 \mathrm{~km} \mathrm{~s}^{-1}$ (Mavromatakis et al. 2002). ROSAT $\mathrm{X}$-ray data infer shock velocities of about $400 \mathrm{~km} \mathrm{~s}^{-1}$ (Schaudel et al. 2002), which implies an age of $2.8 \times 10^{4}$ years.

The Princeton-Arecibo pulsar survey found a pulsar, PSR J1931+30, in the direction of G65.2+5.7 (Camilo et al. 1996). From its dispersion measure of $56 \pm 11 \mathrm{~cm}^{-3} \mathrm{pc}$, a distance of about $3 \mathrm{kpc}$ is inferred, which excludes a physical association with G65.2+5.7. 
Table 1. Observational parameters

\begin{tabular}{lll}
\hline \hline Wavelength & $\lambda 6 \mathrm{~cm}$ & $\lambda 11 \mathrm{~cm}$ \\
Frequency & $4800 \mathrm{MHz}$ & $2639 \mathrm{MHz}$ \\
Bandwidth & $600 \mathrm{MHz}$ & $80 \mathrm{MHz}$ \\
HPBW ['] & 9.5 & 4.4 \\
aperture efficiency[\%] & 62 & 53 \\
beam efficiency[\%] & 67 & 58 \\
$T_{\text {sys }}[\mathrm{K}]$ & 22 & 17 \\
$T_{\mathrm{B}}[\mathrm{K}] / S[\mathrm{Jy}]$ & 0.164 & 2.52 \\
Main Calibrator & $3 \mathrm{C} 286$ & $3 \mathrm{C} 286$ \\
Flux Density & $7.5 \mathrm{Jy}$ & $11.5 \mathrm{Jy}$ \\
Polarization Percentage & $11.3 \%$ & $9.9 \%$ \\
Polarization Angle & $33^{\circ}$ & $33^{\circ}$ \\
No. of coverages & 9 & 7 \\
pixel integration time [s] & 7 & 3.5 \\
rms in TP [mK] & 0.7 & 3.5 \\
rms in PI [mK] & 0.3 & 1.7 \\
\hline
\end{tabular}

The only radio observations of G65.2+5.7 have been those by Reich et al. (1979) at $\lambda 21 \mathrm{~cm}$ and the low-resolution, lowfrequency observations by Kovalenko et al. (1994), reflecting the difficulty in mapping these faint large-diameter objects. With the availability of new sensitive and highly stable receivers, it is now possible to map this object also at higher frequencies with arc minute angular resolution and investigate its spectral properties and magnetic field structure. We describe new sensitive radio measurements in total power and linear polarization at $\lambda 11 \mathrm{~cm}$ and $\lambda 6 \mathrm{~cm}$ of G65.2+5.7 and present results on the spectral and polarization analysis in Sect. 2. A brief discussion of the radio results with respect to observations at other wavelengths is given in Sect. 3, followed by a summary in Sect. 4 .

\section{Observations and result analyses}

Continuum and linear polarization observations of G65.2+5.7 at $\lambda 6 \mathrm{~cm}(4800 \mathrm{MHz})$ were completed with the $25-\mathrm{m}$ telescope at Nanshan station of the Urumqi Observatory. The $\lambda 6 \mathrm{~cm}$ receiver is a copy of a receiver used at the Effelsberg 100-m telescope and was installed in 2004 for the $\lambda 6 \mathrm{~cm}$ polarization survey of the Galactic plane (Sun et al. 2007). Sun et al. (2006) described the performance of the receiving system in some detail. Between 2006 and 2007, nine observations of G65.2+5.7 were completed, which were centered at $\alpha_{2000}=19^{\mathrm{h}} 33^{\mathrm{m}} 6^{\mathrm{s}}$, $\delta_{2000}=31^{\circ} 17^{\prime} 31^{\prime \prime}$. The map size, $\Delta \mathrm{RA} \times \Delta \mathrm{Dec}$, was $5^{\circ} \times 4^{\circ} .5$. The maps were scanned either along the RA- or Dec-direction with a telescope scan velocity of $4^{\prime} / \mathrm{s}$.

The observations of G65.2+5.7 at $\lambda 11 \mathrm{~cm}(2639 \mathrm{MHz})$ were completed with a receiver installed at the secondary focus of the Effelsberg 100-m telescope in 2005. They were carried out in the same way as the observations with the Urumqi telescope. G65.2+5.7 was observed seven times in autumn 2007 mostly in clear sky. In Table 1, we list the parameters of the $\lambda 6 \mathrm{~cm}$ and $\lambda 11 \mathrm{~cm}$ observations with the data of the main calibrator 3C 286 . The $\lambda 11 \mathrm{~cm}$ observations were carried out with a 8-channel narrow band polarimeter. Each channel has a bandwidth of $10 \mathrm{MHz}$. The centre frequencies are separated by $10 \mathrm{MHz}$ and range from $2604 \mathrm{MHz}$ to $2674 \mathrm{MHz}$. The 9th broadband-channel has a bandwidth of $80 \mathrm{MHz}$ and is centered on $2639 \mathrm{MHz}$. This channel was used for all observations in which no narrow band interference was present, which was the case for the observations of G65.2+5.7.

The data processing was completed in exactly the same way as described by Xiao et al. (2008) for the observations of the

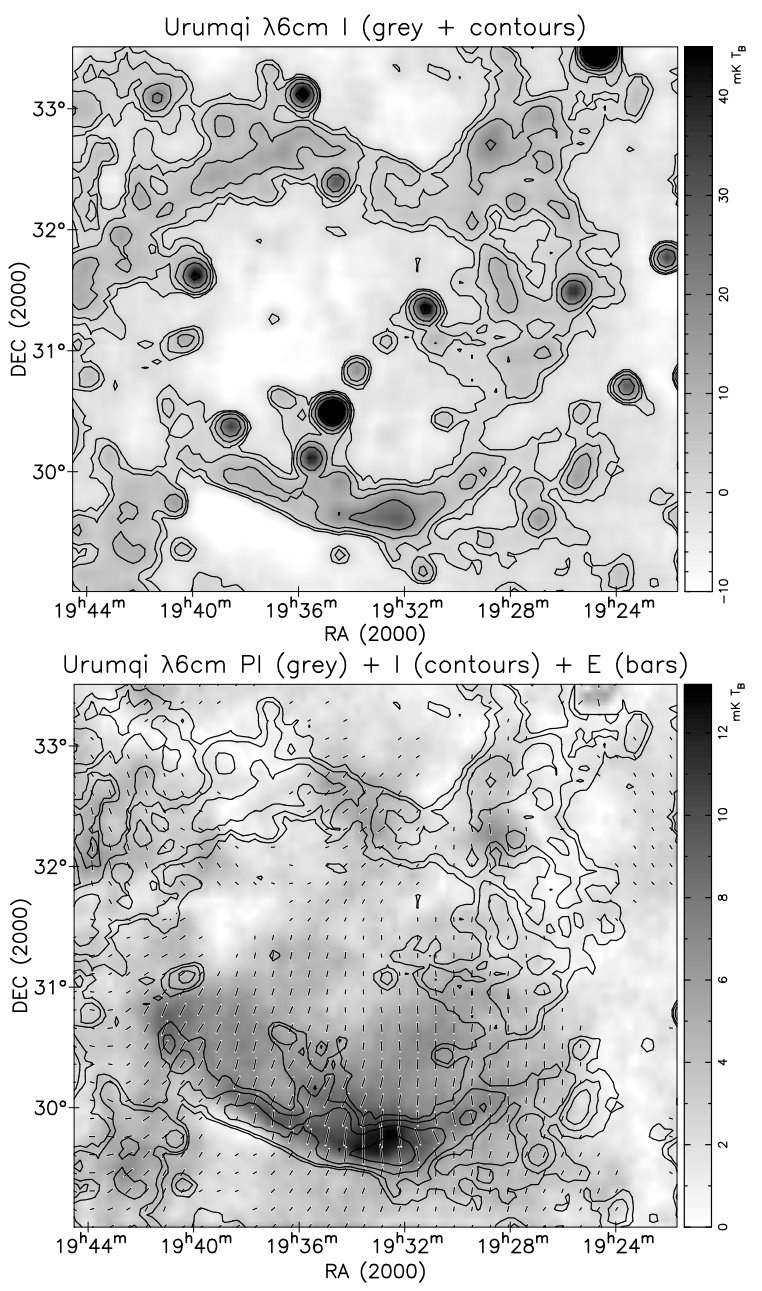

Fig. 1. The Urumqi $\lambda 6 \mathrm{~cm}$ map of G65.2+5.7 with an angular resolution of 9'5. The upper panel shows the distribution of total intensities. The lower panel shows polarized intensities (PI) in grey superimposed on total intensity contours (with point-like sources subtracted) and bars for E-field direction. The length of the bars is proportional to PI. Polarized intensity of $1 \mathrm{mK} T_{\mathrm{B}}$ corresponds to a bar-length of 0 ' 86 . Both panels show total intensity contours starting at $0 \mathrm{mK} T_{\mathrm{B}}$ and increasing $2^{n} \times$ $3 \sigma_{\mathrm{I}}$, where $n=0,1,2,3 \cdots$ and $\sigma_{\mathrm{I}}=0.7 \mathrm{mK} T_{\mathrm{B}}$.

SNR S147, an object of similar size and faintness as G65.2+5.7. In brief, the individual maps for Stokes $I, U$, and $Q$ were checked and low quality data caused by interference were removed. The baselines for Stokes $I$ were corrected by subtracting a second order polynomial fit to the lower emission envelope; this step removed ground emission variations and also the smooth increase in Galactic emission towards the Galactic plane evident in the long scans. We suppressed scanning effects for $U / Q$ maps by applying the "unsharp masking" method described by Sofue \& Reich (1979). Finally, all maps were combined using the PLAIT-algorithm (Emerson \& Gräve 1988).

\subsection{Total intensity and linear polarization maps}

The Urumqi $\lambda 6 \mathrm{~cm}$ total intensity map of G65.2+5.7 and the corresponding polarization intensity map superimposed with vectors in the E-field direction are shown in Fig. 1. From lowintensity areas in the maps, we measured an rms-noise in total intensity and in linear polarized intensity of $0.7 \mathrm{mK} T_{\mathrm{B}}$ and $0.3 \mathrm{mK} T_{\mathrm{B}}$, respectively. The $\lambda 11 \mathrm{~cm}$ maps are shown in Fig. 2. The rms-noise was measured to be $3.5 \mathrm{mK} T_{\mathrm{B}}$ in total 

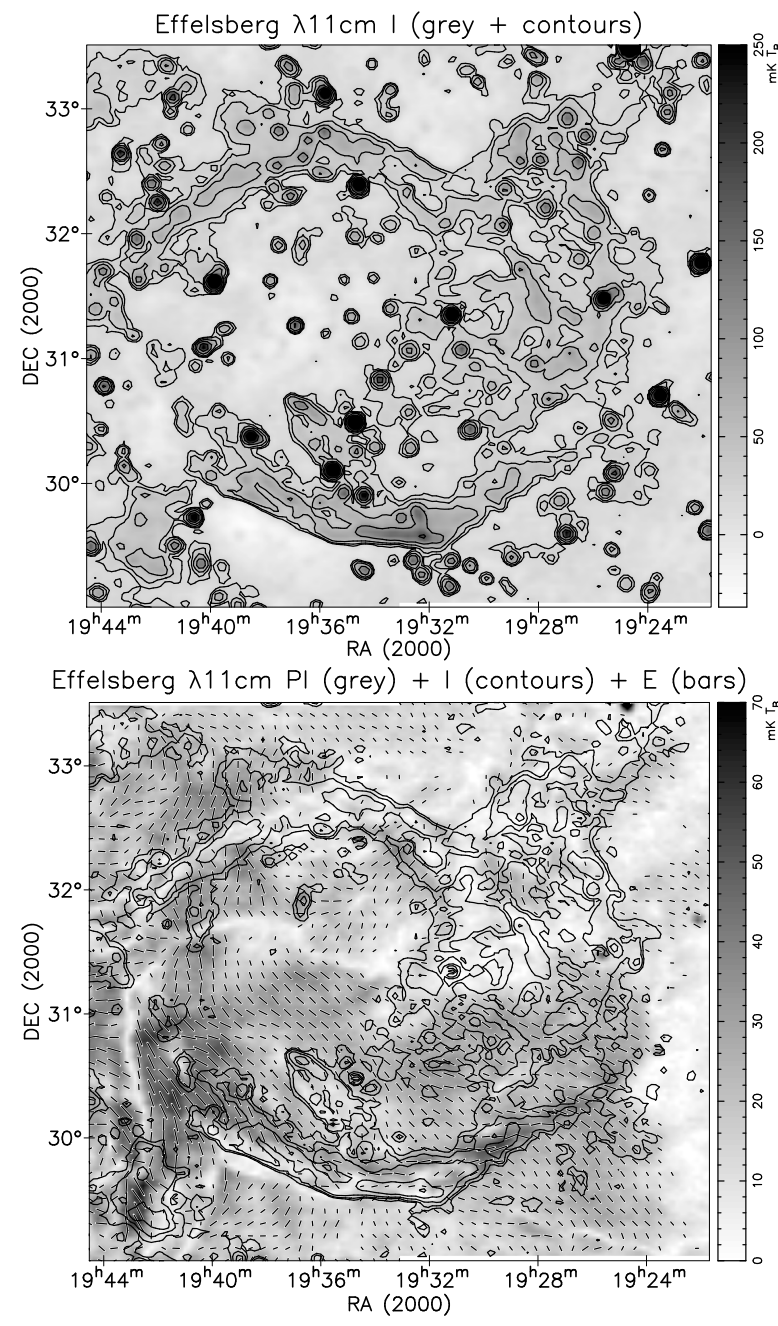

Fig. 2. Same as Fig. 1, but for the Effelsberg $\lambda 11 \mathrm{~cm}$ map with an angular resolution of 4.4. Polarized intensity of $1 \mathrm{mK} T_{\mathrm{B}}$ corresponds to a bar-length of 0.25 . Total intensity contours start at $21 \mathrm{mK} T_{\mathrm{B}}$ and increase in steps of $2^{n} \times 3 \sigma_{\mathrm{I}}$ with $n=0,1,2,3 \cdots$, where $\sigma_{\mathrm{I}}=3.5 \mathrm{mK} T_{\mathrm{B}}$.

intensity and $1.7 \mathrm{mK} T_{\mathrm{B}}$ in linear polarized intensity. Because of the higher angular resolution of the $\lambda 11 \mathrm{~cm}$ map of 4.4, the filamentary elliptical shell is more clearly detected.

For the spectral index analysis we also used an archival $\lambda 21 \mathrm{~cm}$ map obtained with the Effelsberg 100-m telescope in November 1987 at $1408 \mathrm{MHz}$ with a bandwidth of $20 \mathrm{MHz}$ and an angular resolution of 9.4. These observations were made in due course with a $\lambda 21 \mathrm{~cm}$ Galactic plane survey in total intensity (Reich et al. 1990), which covers the plane within an interval of $\pm 4^{\circ}$ in latitude, so that a small part of G65.2+5.7 is included there. Polarization data were taken from a section of the " $\lambda 21 \mathrm{~cm}$ Effelsberg Medium Latitude Survey" (Reich et al. 2004), which was combined with data from the DRAO $1.4 \mathrm{GHz}$ polarization survey (Wolleben et al. 2006) to help ensure detection of any missing large-scale polarized emission. The $\lambda 21 \mathrm{~cm}$ map is shown in Fig. 3.

\subsection{The integrated radio spectrum}

To estimate the integrated flux density of the remnant, we subtracted 20 bright point-like sources by Gaussian fitting within the area of the SNR, and a few just outside, from the $\lambda 11 \mathrm{~cm}$ map and 16 sources from the $\lambda 6 \mathrm{~cm}$ map. The sources are listed

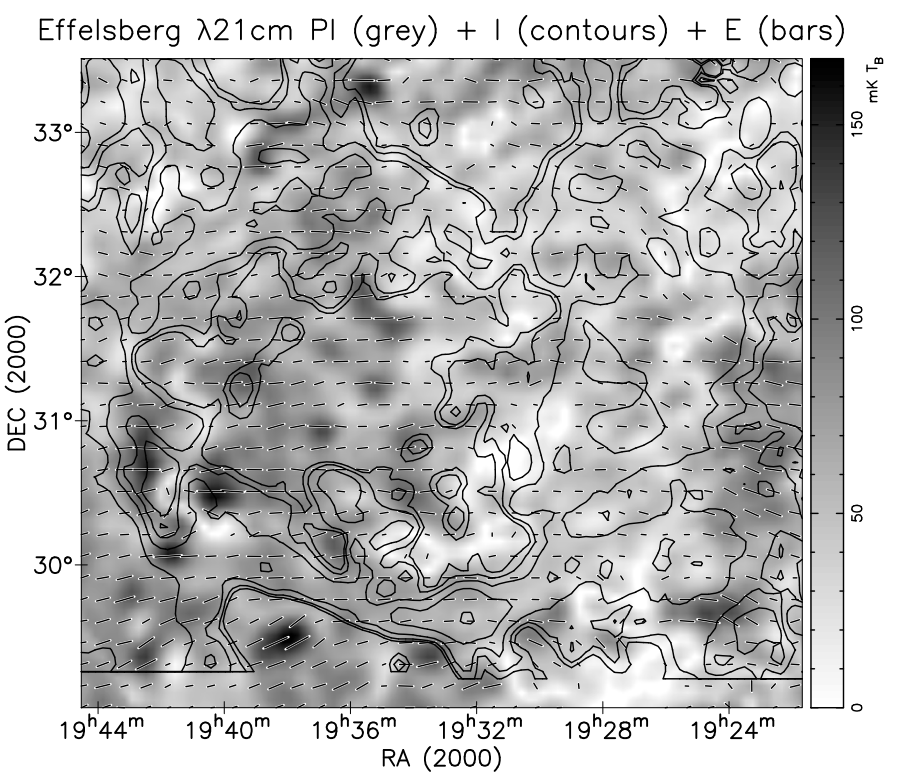

Fig. 3. Effelsberg $\lambda 21 \mathrm{~cm}$ polarized intensity map of G65.2+5.7 with an angular resolution of 9.4. The length of the bars is proportional to PI. Polarized intensity of $1 \mathrm{mK} T_{\mathrm{B}}$ corresponds to a bar-length of 0.075 . Contours show total intensities with compact sources removed starting at $66 \mathrm{mK} T_{\mathrm{B}}$ and increasing in steps of $2^{n} \times 36 \mathrm{mK} T_{\mathrm{B}}$ with $n=0,1,2$, $3 \cdots$.

in Table 2. The position accuracy of the two maps is about $7^{\prime \prime}$ on average, which was estimated by comparing the source positions with those from the NVSS (Condon et al. 1998).

We used the method of temperature-versus-temperature (TT) plot (Turtle et al. 1962) to adjust the base-levels of the entire SNR area at two wavelengths. Both maps were convolved to a common angular resolution of $10^{\prime}$. In agreement with the TT-plot results for three different shell regions (see Sect. 3.3), we found a constant offset of $-6 \mathrm{mK} T_{\mathrm{B}}$ at $\lambda 6 \mathrm{~cm}$. At $\lambda 11 \mathrm{~cm}$, we subtracted a "twisted" surface, which was defined by specific correction values at the four corners $\left(3,3,-6\right.$, and $3 \mathrm{mK} T_{\mathrm{B}}$ from lower left, right to upper left, right) of the map. The integrated flux densities were obtained by integrating the emission enclosed within polygons just outside the periphery of the SNR. From variations outside the SNR, we estimated a remaining uncertainty in the base-level setting of $1 \mathrm{mK} T_{\mathrm{B}}$ at $\lambda 6 \mathrm{~cm}$ and $2 \mathrm{mK} T_{\mathrm{B}}$ at $\lambda 11 \mathrm{~cm}$. By assuming an estimated $5 \%$ calibration uncertainty, we obtained integrated flux densities of $16.8 \pm 1.8 \mathrm{Jy}$ at $\lambda 6 \mathrm{~cm}$ and $21.9 \pm 3.1 \mathrm{Jy}$ at $\lambda 11 \mathrm{~cm}$.

In Fig. 4, these data are plotted together with flux density values from Reich et al. (1979) (408 MHz and $1415 \mathrm{MHz}$ ) and Kovalenko et al. (1994) (83 MHz and $111 \mathrm{MHz}$ ). All data are summarized in Table 3. For the Effelsberg $\lambda 21 \mathrm{~cm}$ map observed in 1987, we obtained the same flux density of $42.4 \pm 4.1 \mathrm{Jy}$ for G65.2+5.7 that was obtained for the map published by Reich et al. (1979). We subtracted the flux densities of pointlike sources listed in Table 2 by assuming a spectral index of $\alpha=-0.79$, which we decided on after caculating the average by weighting the source spectral indices according to their flux densities. The linear fit to the integrated flux densities of G65.2+5.7 as shown in Fig. 4 yields a spectral index of $\alpha=-0.58 \pm 0.07$.

\subsection{TT-plot spectral analysis}

The TT-plot method used for relative zero-level setting in Sect. 2.2 was also used to investigate the spectrum of distinct 
Table 2. Bright sources in the area of SNR G65.2+5.7 fitted from the Effelsberg $\lambda 11 \mathrm{~cm}$ and Urumqi at $\lambda 6 \mathrm{~cm}$ maps, including source positions, flux densities from the NVSS at $\lambda 21 \mathrm{~cm}$, as well as spectral indices. Sources "outside" G65.2+5.7 are listed for completeness.

\begin{tabular}{|c|c|c|c|c|c|c|c|}
\hline & $\begin{array}{c}\text { NVSS } \\
\alpha_{2000}\end{array}$ & $\begin{array}{c}\text { NVSS } \\
\delta_{2000}\end{array}$ & $\begin{array}{l}S_{\mathrm{NVSS}} \\
(\mathrm{mJy})\end{array}$ & $\begin{array}{l}S_{11 \mathrm{~cm}} \\
(\mathrm{mJy})\end{array}$ & $\begin{array}{l}S_{6 \mathrm{~cm}} \\
(\mathrm{mJy})\end{array}$ & $\alpha$ & $\overline{\overline{\text { Comment }}}$ \\
\hline 1 & 192325.26 & 304235.4 & $574.4 \pm 19.1$ & $300 \pm 14$ & $159 \pm 16$ & $-0.83 \pm 0.05$ & \\
\hline 2 & 192513.64 & 303118.9 & $70.3 \pm 2.1$ & $43 \pm 7$ & $31 \pm 5$ & $-0.81 \pm 0.19$ & \\
\hline 3 & 192527.29 & 312923.9 & $384.3 \pm 12.6$ & $222 \pm 12$ & $171 \pm 9$ & $-0.64 \pm 0.07$ & \\
\hline 4 & 192616.04 & 323551.6 & $105.4 \pm 3.9$ & $47 \pm 14$ & & $-1.28 \pm 0.49$ & \\
\hline 5 & 192640.65 & 325655.9 & $67.1 \pm 3.0$ & $48 \pm 12$ & & $-0.53 \pm 0.42$ & 3 unresolved sources \\
\hline 6 & 192656.04 & 293719.6 & $193.2 \pm 6.0$ & $111 \pm 6$ & $67 \pm 17$ & $-0.84 \pm 0.14$ & outside \\
\hline 7 & 192711.53 & 295254.5 & $51.6 \pm 2.2$ & $28 \pm 9$ & $24 \pm 5$ & $-0.77 \pm 0.21$ & outside \\
\hline 8 & 192733.05 & 321401.3 & $79.2 \pm 2.8$ & $52 \pm 9$ & $43 \pm 8$ & $-0.72 \pm 0.18$ & \\
\hline 9 & 193028.65 & 302742.0 & $113.1 \pm 3.4$ & $71 \pm 4$ & $37 \pm 4$ & $-1.03 \pm 0.15$ & \\
\hline 10 & 193046.84 & 310559.1 & $126.2 \pm 4.4$ & $62 \pm 8$ & $43 \pm 9$ & $-0.80 \pm 0.15$ & \\
\hline 11 & 193108.60 & 312233.5 & $428.6 \pm 12.9$ & $311 \pm 11$ & $232 \pm 14$ & $-0.63 \pm 0.05$ & $11 / 6 \mathrm{~cm}$ fit \\
\hline 12 & 193114.43 & 291235.2 & $67.7 \pm 2.1$ & $59 \pm 16$ & $55 \pm 5$ & $-0.66 \pm 0.19$ & outsic \\
\hline 13 & 193245.08 & 310610.7 & $35.8 \pm 1.1$ & $38 \pm 8$ & & $0.09 \pm 0.35$ & \\
\hline 14 & 193348.95 & 305145.1 & $64.7 \pm 2.0$ & $90 \pm 15$ & $98 \pm 8$ & $0.14 \pm 0.07$ & $11 / 6 \mathrm{~cm}$ fit \\
\hline 15 & 193419.84 & 292007.4 & $65.5 \pm 2.3$ & $59 \pm 9$ & $37 \pm 8$ & $-0.84 \pm 0.20$ & atsi \\
\hline 16 & 193438.47 & 322447.8 & $625.7 \pm 18.8$ & $299 \pm 13$ & $146 \pm 13$ & $-0.80 \pm 0.04$ & \\
\hline 17 & 193445.20 & 303058.8 & $235.2 \pm 7.1$ & $469 \pm 14$ & $579 \pm 23$ & $0.35 \pm 0.11$ & \\
\hline 18 & 193534.24 & 300800.9 & $688.7 \pm 23.8$ & $359 \pm 43$ & $183 \pm 22$ & $-0.81 \pm 0.04$ & \\
\hline 19 & 193556.39 & 330822.0 & $77.0 \pm 2.3$ & $216 \pm 10$ & $250 \pm 16$ & $0.24 \pm 0.18$ & outside, $11 / 6 \mathrm{~cm}$ fit \\
\hline 20 & 193658.60 & 311725.8 & $149.7 \pm 4.5$ & $93 \pm 9$ & $55 \pm 14$ & $-0.89 \pm 0.16$ & \\
\hline 21 & 193838.31 & 302355.4 & $92.6 \pm 2.8$ & $172 \pm 15$ & $171 \pm 18$ & $-0.01 \pm 0.29$ & $11 / 6 \mathrm{~cm} \mathrm{fit}$ \\
\hline 22 & 194003.36 & 313740.6 & $538.5 \pm 18.4$ & $353 \pm 15$ & $220 \pm 17$ & $-0.77 \pm 0.05$ & \\
\hline 23 & 194026.13 & 310623.9 & $199.4 \pm 6.0$ & $112 \pm 15$ & $61 \pm 8$ & $-0.94 \pm 0.14$ & \\
\hline 24 & 194039.90 & 294446.8 & $300.1 \pm 10.6$ & $147 \pm 9$ & $73 \pm 17$ & $-0.92 \pm 0.11$ & outside \\
\hline 25 & 194214.83 & 321538.7 & $177.2 \pm 5.3$ & $98 \pm 12$ & & $-0.93 \pm 0.23$ & \\
\hline 26 & 194229.66 & 322446.9 & $83.3 \pm 2.5$ & $58 \pm 12$ & & $-0.58 \pm 0.35$ & outside \\
\hline 27 & 194300.76 & 315814.2 & $76.3 \pm 2.3$ & $60 \pm 10$ & $43 \pm 16$ & $-0.79 \pm 0.20$ & \\
\hline 28 & 194405.01 & 304645.8 & $156.6 \pm 4.7$ & $82 \pm 4$ & $37 \pm 12$ & $-0.96 \pm 0.16$ & outside \\
\hline
\end{tabular}

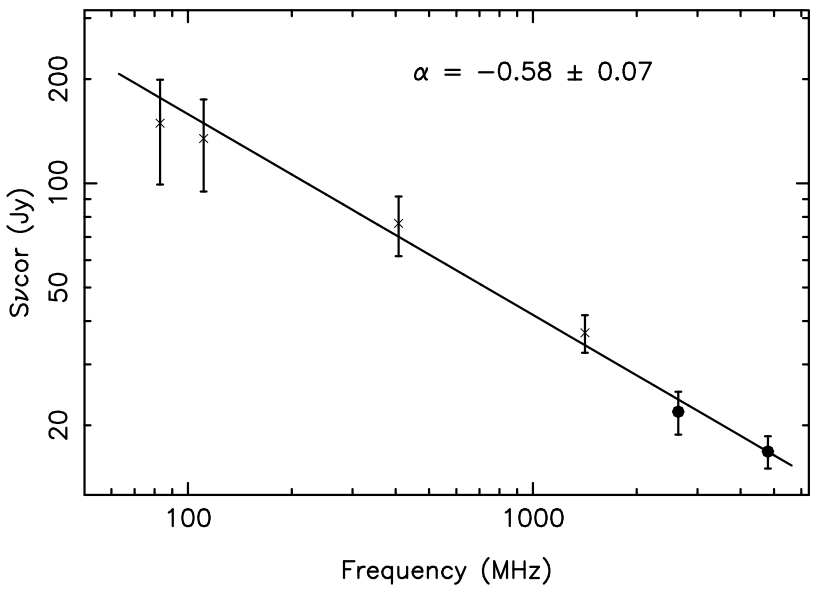

Fig. 4. Spectrum of the integrated radio flux densities of G65.2+5.7. The spectral index is $\alpha=-0.58 \pm 0.07$. All flux densities were corrected for the contribution of compact sources (Table 3 ).

emission structures in a way independent of a consistent baselevel setting of both maps. Spectral index variations of structures within a source could then be recognized or the integrated spectrum checked. We smoothed both maps to $10^{\prime}$ and applied this method for three different sections of the G65.2+5.7 shell, which are marked in Fig. 6. The results are shown in Fig. 5 for the $\lambda 11 \mathrm{~cm} / \lambda 6 \mathrm{~cm}$ data. A clear temperature-temperature relation is evident in all cases. The same TT-plot procedure was used for the $\lambda 21 \mathrm{~cm} / \lambda 6 \mathrm{~cm}$ data. The temperature spectral index $\beta(=\alpha-2)$ found from fitting the slope is $\beta_{11 / 6}=-2.45 \pm 0.10$ and $\beta_{21 / 6}=-2.61 \pm 0.24$ for the southern shell, $\beta_{11 / 6}=-2.46 \pm 0.24$ and $\beta_{21 / 6}=-2.65 \pm 0.27$ for the northeastern shell, and $\beta_{11 / 6}=$ $-2.54 \pm 0.44$ and $\beta_{21 / 6}=-2.71 \pm 0.45$ for the western shell. The error in $\beta$ for the diffuse western part of the shell region is large, probably due to its weak emission and confusion with weak unresolved background sources, which could not be subtracted. All values agree with both each other and the spectral index of the integrated spectrum to within the errors. However, we always obtain somewhat steeper spectra for the $\lambda 21 \mathrm{~cm} / \lambda 6 \mathrm{~cm}$ data than the $\lambda 11 \mathrm{~cm} / \lambda 6 \mathrm{~cm}$ data, which reflects the slightly lower integrated flux density at $\lambda 11 \mathrm{~cm}$ relative to the fit shown in Fig. 4 and the marginally higher value we obtained at $\lambda 21 \mathrm{~cm}$.

\subsection{Spectral index map}

The Urumqi $\lambda 6 \mathrm{~cm}$ and the Effelsberg $\lambda 11 \mathrm{~cm}$ maps with pointlike sources removed and base-level corrected were both convolved to a common angular resolution of $10^{\prime}$. We calculated the spectral index for each pixel from the brightness temperatures at the two frequencies. We defined a lower intensity limit of $4 \mathrm{mK} T_{\mathrm{B}}$ and $12 \mathrm{mK} T_{\mathrm{B}}$ for the $\lambda 6 \mathrm{~cm}$ and $\lambda 11 \mathrm{~cm}$ map, respectively, to achieve reasonable spectral indices without the influence of noise and local distortions. We display the spectral index map between $\lambda 6 \mathrm{~cm}$ and $\lambda 11 \mathrm{~cm}$ in Fig. 6 . Possible remaining variations in the base-levels at $\lambda 11 \mathrm{~cm}$ or $\lambda 6 \mathrm{~cm}$ cause a systematic uncertainty in the spectral indices of $\Delta \beta \sim 0.2$. The uncertainty is larger when total intensities are lower.

The filament emerging from the southern shell towards the north has a somewhat steeper spectrum than that of the southern shell. This is confirmed by $\beta_{11 / 6}=-2.75 \pm 0.28$ obtained from TT-plot, although this spectral index value is within the errors of those obtained for the other shell regions. 
Table 3. Integrated flux densities of G65.2+5.7, before $\left(S_{\nu}\right)$ and after $\left(S_{\nu}\right.$ cor $)$ subtraction of point-like sources within the boundary of SNR.

\begin{tabular}{rcccl}
\hline \hline $\begin{array}{r}v \\
(\mathrm{MHz})\end{array}$ & $\begin{array}{c}\text { Resolution } \\
(\operatorname{arcmin})\end{array}$ & $\begin{array}{c}S_{v} \\
(\mathrm{Jy})\end{array}$ & $\begin{array}{c}S_{v} \text { cor } \\
(\mathrm{Jy})\end{array}$ & Ref. \\
\hline 83 & $310 \times 240$ & $200.0 \pm 50$ & $149.2 \pm 50$ & Kovalenko et al. (1994) \\
111 & $310 \times 240$ & $175.0 \pm 40$ & $134.7 \pm 40$ & Kovalenko et al. (1994) \\
408 & 37 & $91.0 \pm 15$ & $76.6 \pm 15$ & Reich et al. (1979) \\
1415 & 9 & $42.4 \pm 4.6$ & $37.0 \pm 4.6$ & Reich et al. (1979) \\
2639 & 4.4 & & $21.9 \pm 3.1$ & this paper \\
4800 & 9.5 & & $16.8 \pm 1.8$ & this paper \\
\hline
\end{tabular}
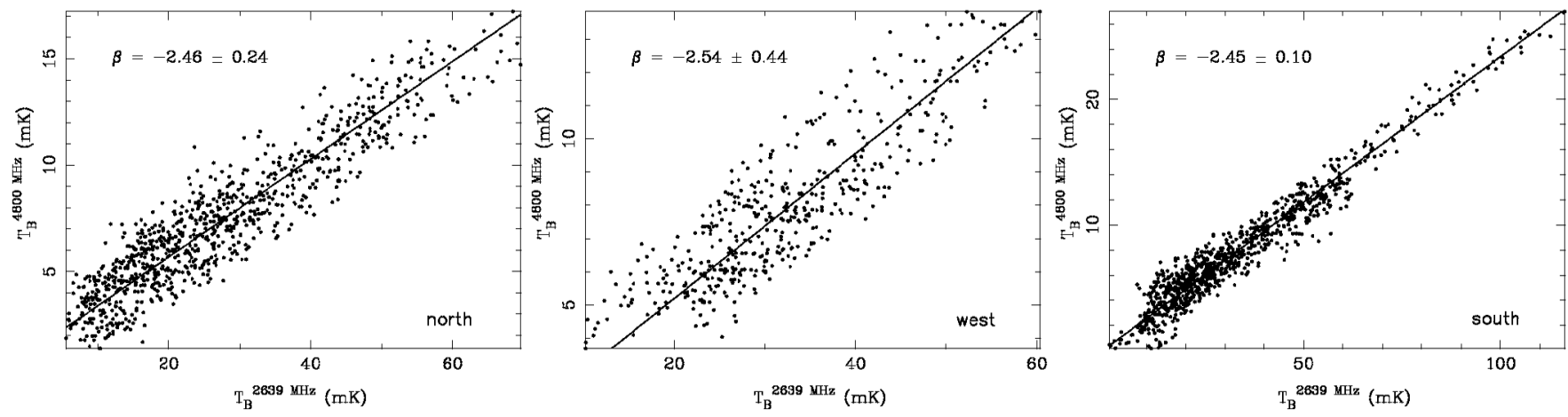

Fig. 5. T-T plots for different shell sections of G65.2+5.7 between $\lambda 11 \mathrm{~cm}$ and $\lambda 6 \mathrm{~cm}$.

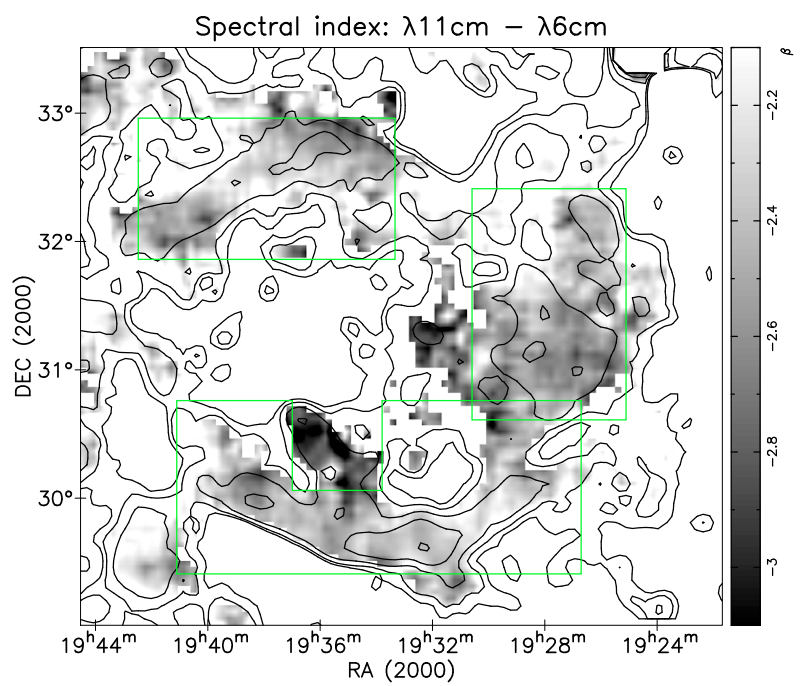

Fig. 6. Spectral index map calculated between $\lambda 11 \mathrm{~cm}$ and $\lambda 6 \mathrm{~cm}$ at $10^{\prime}$ angular resolution for total intensities exceeding $12 \mathrm{mK} T_{\mathrm{B}}$ and $4 \mathrm{mK} T_{\mathrm{B}}$ at $\lambda 11 \mathrm{~cm}$ and $\lambda 6 \mathrm{~cm}$, respectively. The overlaid contours show total intensities at $\lambda 11 \mathrm{~cm}$ starting at $12 \mathrm{mK} T_{\mathrm{B}}$, increasing in steps of $2^{\mathrm{n}} \times 3 \sigma_{\mathrm{I}}$, where $n=0,1,2,3 \cdots$, and $\sigma_{\mathrm{I}}=2.2 \mathrm{mK} T_{\mathrm{B}}$. The regions used for TT-plots in Fig. 5 are indicated by boxes.

Reich et al. (1979) found an indication of spectral flattening towards the centre of G65.2+5.7 of about $\Delta \beta \sim 0.3$ when comparing their Effelsberg $\lambda 21 \mathrm{~cm}$ map with low-resolution data at $408 \mathrm{MHz}$ (Haslam et al. 1974). This finding cannot be verified by the present data. The emission in the central area is too faint at higher frequencies to derive meaningful spectral indices in relation to the base-level uncertainties.

\subsection{The linear polarization properties of $G 65.2+5.7$}

The $\lambda 6 \mathrm{~cm}$ and $\lambda 11 \mathrm{~cm}$ polarization maps shown in the lower panels of Figs. 1 and 2 indicate the similar polarization features at the two frequencies. Four polarized patches appear at $\lambda 6 \mathrm{~cm}$, three smaller and fainter ones located in the northern area of G65.2+5.7, and a single large patch covering the southern shell and part of the inner area of the SNR. Apart from the enhanced polarization along the SNR southern shell, most of the polarization structures might originate from polarized diffuse interstellar emission possibly mixed with polarized emission from the SNR of about similar strength. We note low polarized emission along the northern shell at both wavelengths.

As shown in Fig. 3 the $\lambda 21 \mathrm{~cm}$ polarization data do not indicate any polarized emission at all compared to G65.2+5.7, since neither the polarized intensity nor the polarization vectors change significantly in the direction of the SNR relative to its surroundings. This indicates that most of the polarized emission seen in this area at $\lambda 21 \mathrm{~cm}$ originates from the foreground within the distance to G65.2+5.7 of about $800 \mathrm{pc}$.

The large-scale emission components are missing in the two polarization maps at $\lambda 6 \mathrm{~cm}$ and $\lambda 11 \mathrm{~cm}$, because the end-points of each scan in the individual $U$ and $Q$ maps were set to zero during data processing. This limits the interpretation of polarization structures (Reich 2006), except for the strong polarized region along the southern shell at $\lambda 6 \mathrm{~cm}$.

Following Sun et al. (2007), we used the WMAP polarization data at $22.8 \mathrm{GHz}$ (Page et al. 2007) to recover missing largescale structures at $\lambda 6 \mathrm{~cm}$. There is no signature of G65.2+5.7 in the Stokes $U$ and $Q$ maps at $22.8 \mathrm{GHz}$. The convolved $22.8 \mathrm{GHz}$ PI map shows a smooth intensity increase below Galactic latitudes of about 5.5 . Above 5.5 , the orientation of the polarization $B$-vectors is less regular (Fig. 7). We convolved the WMAP $22.8 \mathrm{GHz} U$ and $Q$ maps corresponding to an angular resolution of $2^{\circ}$ and scaled them to $4.8 \mathrm{GHz}(\lambda 6 \mathrm{~cm})$ assuming a temperature spectral index of $\beta=-2.65$, which was taken from the spectral index map of total intensities between $408 \mathrm{MHz}$ and $1420 \mathrm{MHz}$ by Reich \& Reich (1988). We assume the same spectral index for the Galactic diffuse polarized emission. The difference between the scaled WMAP data and the $\lambda 6 \mathrm{~cm}$ maps, when convolved to the same beam size of $2^{\circ}$, results in an offset of $-0.4 \mathrm{mK} T_{\mathrm{B}}$ for Stokes $U$ and $-2.3 \mathrm{mK} T_{\mathrm{B}}$ for Stokes $Q$, which 


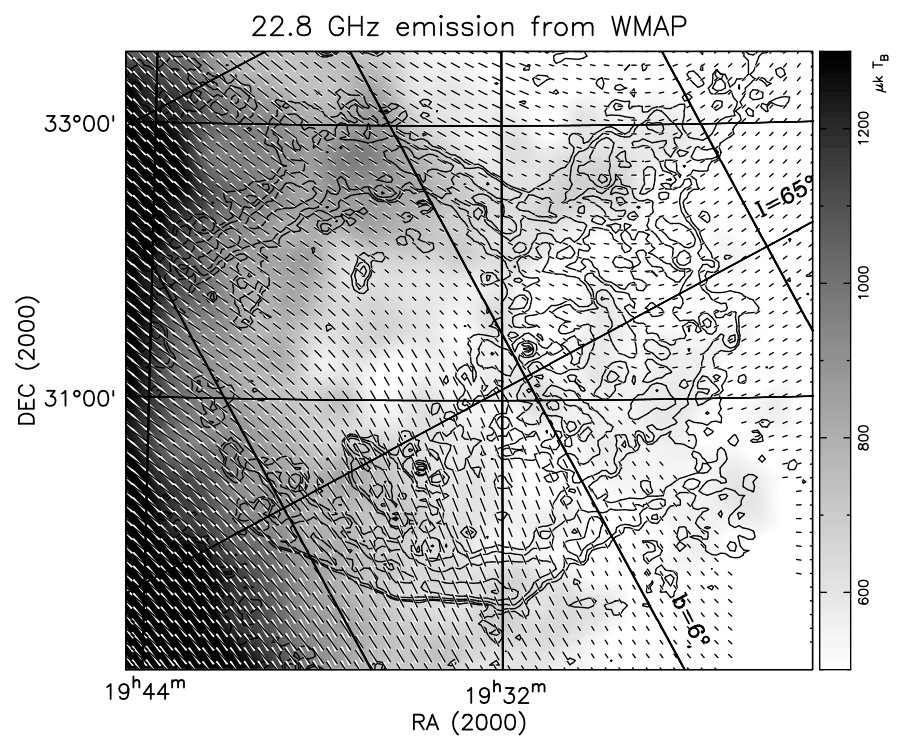

Fig. 7. The WMAP $22.8 \mathrm{GHz}$ map. The total intensity emission (greyscale) map is smoothed to an angular resolution of $2^{\circ}$ with polarization $B$-vectors overlaid. Their length is proportional to the polarized intensity. The overlaid contours show total intensities at $\lambda 11 \mathrm{~cm}$ at the same level as in Fig. 2 with compact sources removed.

is equivalent to $2.3 \mathrm{mK} T_{\mathrm{B}}$ of polarized intensity. The offsets were added to the original $\lambda 6 \mathrm{~cm} U$ and $Q$ data. The polarized intensity map at $\lambda 6 \mathrm{~cm}$ derived from the restored data is shown in Fig. 8.

We then compared the $\lambda 6 \mathrm{~cm}$ polarization maps with and without absolute zero-level restoration. We found in general that the addition of missing large-scale polarization did not change the morphology of the observed polarization at $\lambda 6 \mathrm{~cm}$ significantly. The polarized signal from the southern shell of G65.2+5.7 was strong enough to remain almost unchanged when adding the large-scale emission component. Strong polarization emission of the diffuse interstellar medium near to the Galactic plane in the south-eastern area of the map is unrelated to G65.2+5.7. However, we note some changes in areas of lower polarized emission. For instance, the polarized emisssion is more pronounced in the direction of the extended HII-region LBN 150, also known as Sh2-96, $\left(19^{\mathrm{h}} 28.7^{\mathrm{m}},+32^{\circ} 41^{\prime}, \mathrm{J} 2000\right)$ in Fig. 8, which itself does not emit polarized emission, but may act as a Faraday screen by rotating polarized emission from larger distances.

The procedure for recovering missing large-scale emission by using the WMAP polarization data, needs to be modified in case the Faraday rotation is too large or becomes significant at longer wavelengths because of the $\lambda^{2}$-dependence of the polarization angle for a given RM. Therefore, it seems questionable that the $\lambda 11 \mathrm{~cm} U$ and $Q$ maps should by corrected in the same way as the $\lambda 6 \mathrm{~cm}$ polarization data. The level of Galactic PI at $\lambda 11 \mathrm{~cm}$, however, is calculated to be about $11.5 \mathrm{mK} T_{\mathrm{B}}$. This large-scale polarized signal needs to be compared with the observed polarized signals at $\lambda 11 \mathrm{~cm}$, which are only three times stronger on the inner side of the southern shell and only twice as strong for the average polarization of the entire SNR. We conclude that the missing large-scale polarization is a serious contamination of the $\lambda 11 \mathrm{~cm}$ data and any RMs calculated from the $\lambda 11 \mathrm{~cm}$ and the $\lambda 6 \mathrm{~cm}$ data will by affected by an unknown amount by that effect.

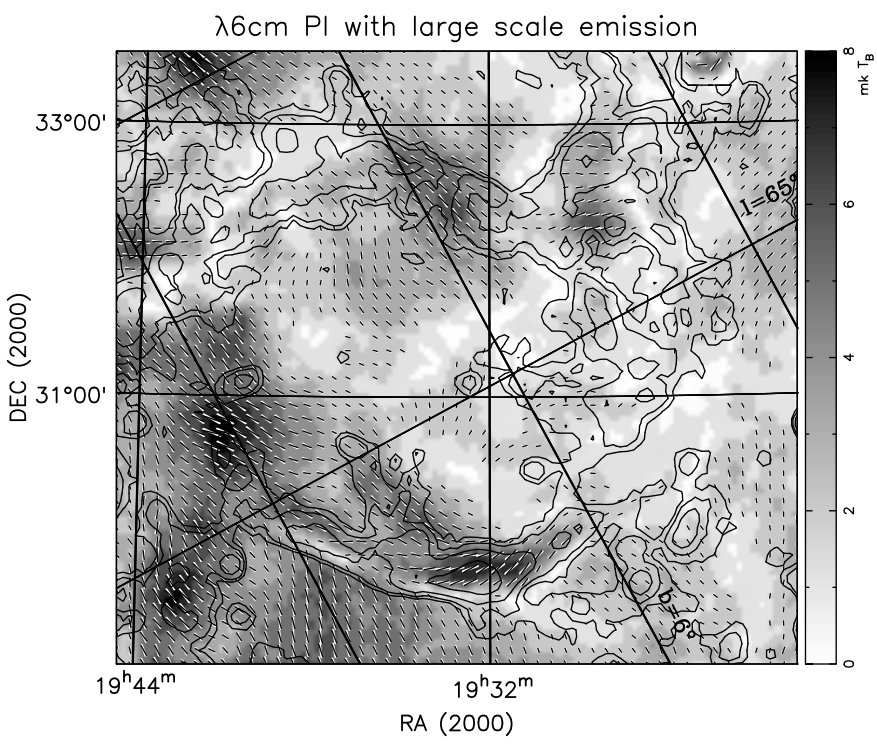

Fig. 8. The $\lambda 6 \mathrm{~cm}$ polarized intensity map (grey scale) with large-scale polarized emission restored from WMAP $22.8 \mathrm{GHz}$ polarization data. B-field vectors are shown for the case of negligible Faraday rotation. Contours show $\lambda 6 \mathrm{~cm}$ intensities as in Fig. 1.

\subsection{The northern area of $G 65.2+5.7$}

The polarized emission seen in the northern area of the SNR is much weaker than in the southern area and not very clearly related to the SNR shell. Along the northern shell, depolarization at both $\lambda 11 \mathrm{~cm}$ and $\lambda 6 \mathrm{~cm}$ is evident, which may have various causes. Firstly, background polarization is depolarized by small-scale magneto-ionic fluctuations in the shell; secondly, polarized emission from the SNR shell has a different orientation and cancels, at least partly, the background emission; or, thirdly, Faraday rotation in the shell rotates background polarization in such a way that it cancels the foreground polarization. A combination of the different scenarios is certainly possible. We note that Mavromatakis et al. (2002) measured the highest thermal electron densities for the optical filaments in the northern shell, which may explain the high depolarization. The low percentage polarization in the northern area, compared to that of the southern shell, may indicate a less regular magnetic field. We conclude that the magnetic field properties along the northern shell cannot be determined reliably from the data available to us.

\subsection{The southern shell of G65.2+5.7}

As shown in Fig. 9 the polarized emission at $\lambda 6 \mathrm{~cm}$ reaches its maximum at the southern shell. The polarization percentage of the southern shell locally is as high as $54 \%$ at $\lambda 6 \mathrm{~cm}$. Lower polarized emission at $\lambda 11 \mathrm{~cm}$ at the same position indicates strong depolarization at this wavelength. Inside the shell the polarization percentage at $\lambda 11 \mathrm{~cm}$ is about $38 \%$.

As expected for an evolved SNR, a well ordered magnetic field is detected in the shell. Rotating the polarization $E$-vectors shown in Fig. 1 by $90^{\circ}$ results in an observed magnetic field direction that is almost tangential to the shell (Fig. 8). Thus, the magnetic field intrinsic to the shell is tangential when the Faraday rotation is low. If we accept the deviations (of about $15^{\circ}$ ) from tangential orientations as the result of intrinsic plus foreground RMs, then the total RM should not exceed about $70 \mathrm{rad} \mathrm{m}^{-2}$. 


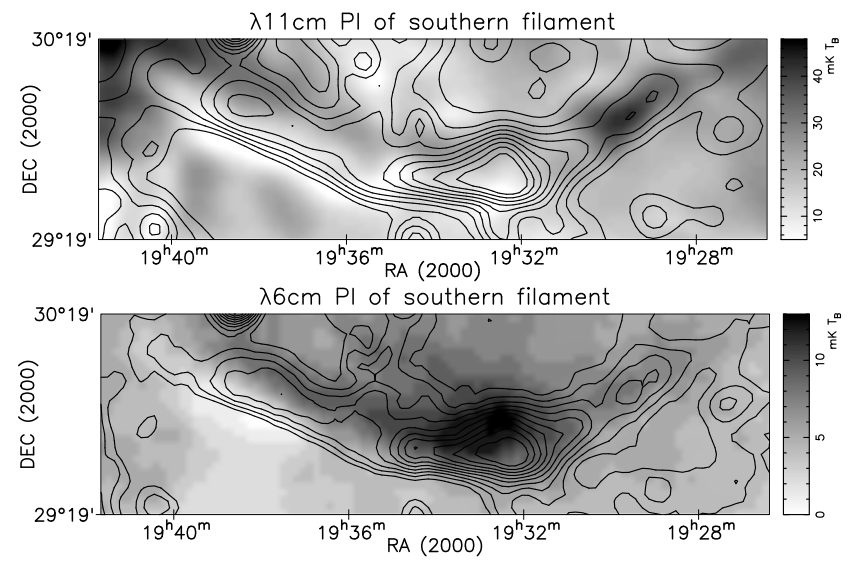

Fig. 9. The observed polarized intensities in grey scales for the southern shell at $\lambda 11 \mathrm{~cm}$ and $\lambda 6 \mathrm{~cm}$ overlaid with contours of corresponding total intensities. Both maps are smoothed to $10^{\prime}$ angular resolution.

We estimated the contribution of RM from the foreground interstellar medium. The NE2001 model of Cordes \& Lazio (2002) predicts a dispersion measure of DM $=9 \mathrm{pc} \mathrm{cm}^{-3}$ in the direction of G65.2+5.7. The magnetic field strength along the lightof-sight, $B_{\|}$, is about $\sim 2 \mu \mathrm{G}$, which is a typical value for the local regular magnetic field (Han et al. 2006). Then RM is calculated by $\mathrm{RM}=0.81 B_{\|} \mathrm{DM}$ resulting in about $-14 \mathrm{rad} \mathrm{m}^{-2}$. This Galactic foreground RM has little effect (only a few degrees) on the polarization vector orientation measured at $\lambda 6 \mathrm{~cm}$.

When rotating the $E$-vectors at $\lambda 11 \mathrm{~cm}$ in the same way as at $\lambda 6 \mathrm{~cm}$, magnetic directions that differ up to $80^{\circ}$ from a tangential orientation direction imply values of RM of the order of $150 \mathrm{rad} \mathrm{m}^{-2}$ for the $\lambda 11 \mathrm{~cm}$ data to agree with a tangential magnetic field direction. This large RM disagrees with the $\lambda 6 \mathrm{~cm}$ data. However, as stated above, the observed level of the $\lambda 11 \mathrm{~cm}$ polarized emission is too low to be unaffected by unrelated Galactic large-scale emission. This needs to be taken into account before calculating any RM for G65.2+5.7, but we have insufficient information to do this. Clearly, we require observations at wavelengths shorter than $\lambda 6 \mathrm{~cm}$ of sufficiently high angular resolution to calculate the RM properties of G65.2+5.7 in combination with our $\lambda 6 \mathrm{~cm}$ data and thus contain the suggested tangential magnetic field orientation along its southern shell.

We can estimate the magnetic field strength in the southern shell by assuming energy equipartition between the magnetic field and electrons and protons in the SNR-shell using the formula by Fürst \& Reich (2004), where the radius of a source is replaced by the volume $\mathrm{V}$ to deal with a shell section

$B_{\min } \approx 175 \cdot \Phi^{-2 / 7} \cdot V\left(\mathrm{pc}^{3}\right)^{-2 / 7} \cdot d(\mathrm{kpc})^{4 / 7} \cdot S_{1 \mathrm{GHz}}(\mathrm{Jy})^{2 / 7}$.

We extrapolated the integrated $\lambda 6 \mathrm{~cm}$ flux density without compact sources for the southern shell section centred on $\alpha_{2000}=$ $19^{\mathrm{h}} 33^{\mathrm{m}}, \delta_{2000}=30^{\circ}\left(4^{\circ}\right.$ in $\alpha, 1^{\circ}$ in $\left.\delta\right)$ of $4.0 \mathrm{Jy}$ to $8.8 \mathrm{Jy}$ at $1 \mathrm{GHz}$ for the error-weighted mean spectral index from TT-plots corresponding to $\alpha=-0.50$. We estimated the thickness of the southern shell from our highest angular resolution data at $\lambda 11 \mathrm{~cm}$ to be about $10 \%$ of the radius, or about $2.3 \mathrm{pc}$. For a distance of $0.8 \mathrm{kpc}$, we calculated a volume of the southern shell region of about $6500 \mathrm{pc}^{3}$. The estimated equipartition magnetic field $B_{\min }$ is about $23 \mu \mathrm{G}$ for a filling factor $\Phi=1$, although this is a lower limit. In case of a highly filamentary shell, such as G65.2+5.7, a filling factor of $\Phi=0.1$ may be more appropriate, which would increase $B_{\min }$ to $45 \mu \mathrm{G}$.
Another approach to estimating equipartition magnetic fields in SNRs was described by Foster (2005). This method results in a magnetic field strength of $B \sim 36 \mu \mathrm{G}$ for $\alpha=-0.50$ over a frequency range of $v_{\min , \max }=0.1-100 \mathrm{GHz}$, a line-of-sight extension of the radio-emitting region of $50 \mathrm{pc}$, and a radio intensity of the southern shell of $I_{v}=0.2 \times 10^{-18} \mathrm{erg} \mathrm{s}^{-1} \mathrm{~cm}^{-2} \mathrm{~Hz}^{-1} \mathrm{sr}^{-1}$. We conclude that the magnetic field of the southern shell has a strength of between 20 and $50 \mu \mathrm{G}$.

\section{Discussion}

The radio properties of G65.2+5.7 show a number of similarities with another bilateral SNR G156.2+5.7 (Reich et al. 1992; Xu et al. 2007), which also has the lowest surface brightness of all known SNRs with a surface brightness of $\Sigma_{1 \mathrm{GHz}}=$ $5.8 \times 10^{-23} \mathrm{Wm}^{-2} \mathrm{~Hz}^{-1} \mathrm{sr}^{-1}$ at $1 \mathrm{GHz}$. The surface brightness of G65.2+5.7 is about $\Sigma_{1 \mathrm{GHz}}=1.3 \times 10^{-22} \mathrm{Wm}^{-2} \mathrm{~Hz}^{-1} \mathrm{sr}^{-1}$, just about twice that of G156.2+5.7. Both SNRs are located outside the Galactic plane and at a similar distance of $800 \mathrm{pc}$ or $1 \mathrm{kpc}$, although the shape of G65.2.+5.7 is more elliptical and its size is about 50\% larger, possibly indicating that it is a more evolved object. Both are very bright in X-rays. The symmetry axis of both objects shows a large inclination relative to the Galactic plane, which has been taken as evidence that the ambient magnetic field has a similar inclination. The WMAP $22.8 \mathrm{GHz}$ polarization data at an angular resolution of $2^{\circ}$ in Fig. 7 indicate that the magnetic field is aligned almost parallel to the Galactic plane up to about 6.5 latitude. Above 6.5 latitude, the direction is less clearly defined. Of course, the WMAP data show the integrated polarization along the line-of-sight and local deviations in the magnetic field might be possible.

Some differences between G65.2+5.7 and G156.2+5.7 exist in their polarization properties. At $\lambda 6 \mathrm{~cm}$ and $\lambda 11 \mathrm{~cm}$ $\mathrm{G} 156.2+5.7$ has a higher fractional polarization. Depolarization along the outer shell of G65.2+5.7 seems to support the classification of a "thermal composite", based on X-ray data, where a dense outer shell causes absorption of X-ray emission and strong depolarization (Shelton et al. 2004).

Orlando et al. (2007) presented simulations of the bilateral morphology of SNRs caused by density gradients in the interstellar medium or a gradient in the ambient magnetic field perpendicular to the radio shell. Smooth gradients are probably a simplification of the true conditions. However, we note that the intensity differences between the two arcs of the shell of G65.2+5.7 seem to agree with some of the simulations by Orlando et al. (2007, their Fig. 7, panels A and B), in which quasi-parallel particle injection was assumed and the viewing angle was perpendicular to both $\langle B\rangle$ and the gradients of either density or magnetic field strength. Slanting similar radio arcs were produced if the gradient was perpendicular to $\langle B\rangle$. In the quasi-perpendicular case, Orlando et al. (2007) found a bilateral shape when the gradient of the ambient $\langle B\rangle$ or density was parallel to $\langle B\rangle$. The symmetry axis of the SNR is always aligned with the gradient of density or the magnetic field. As indicated by the WMAP total intensity map (Fig. 7) or $\mathrm{H}_{\alpha}$ data (Fig. 13, right panel), thermal emission is evident along the northern and southern shell, and more smooth in the north than in the south. In addition to the general density gradient in the direction of Galactic latitude, this indicates a density difference in the direction of longitude, which results in compression differences in the interacting SNR shell and to a brightness difference as observed. 

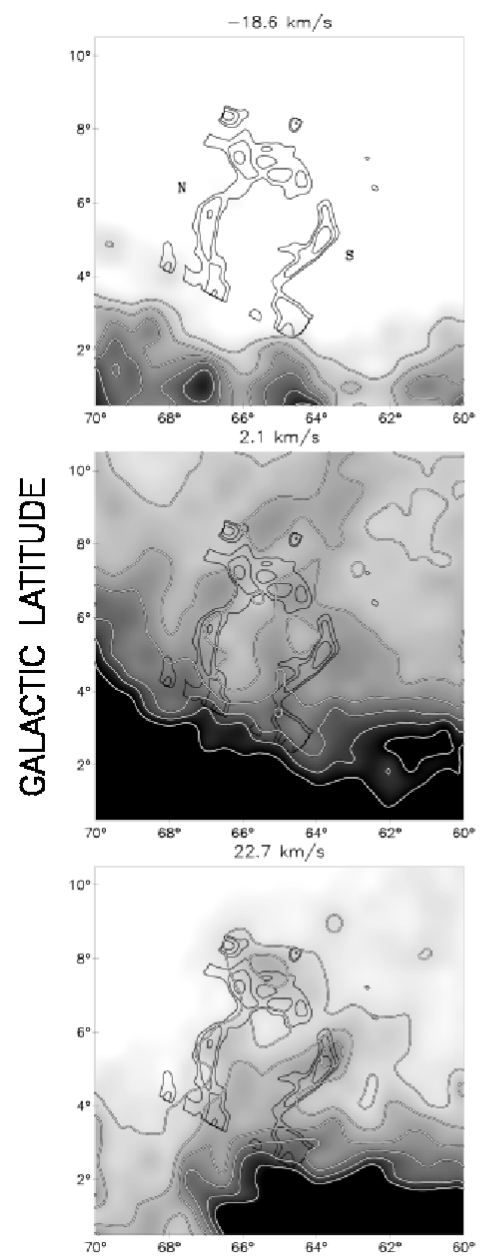
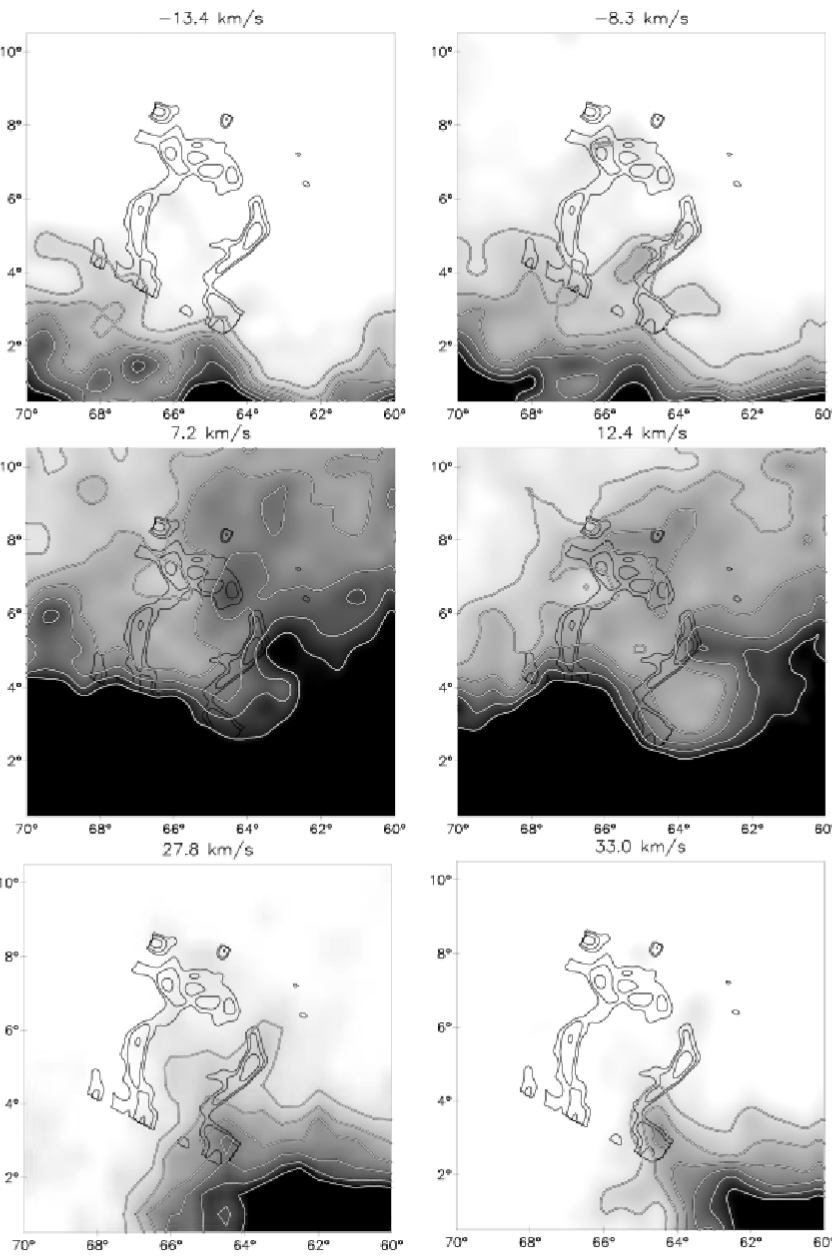

GALACTIC LONGITUDE

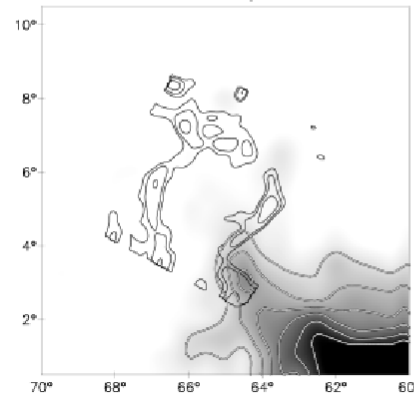

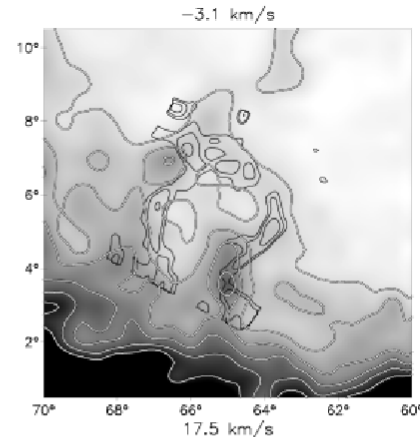
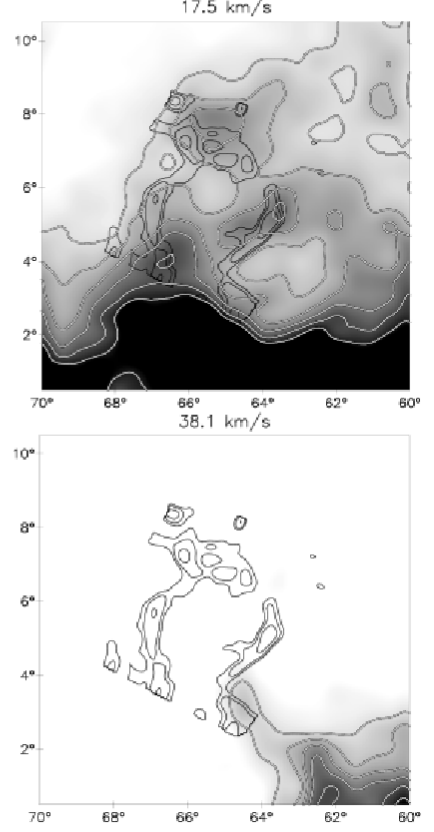

Fig. 10. The $\lambda 21 \mathrm{~cm}$ HI-intensities in the area of G65.2+5.7 for the velocity range from $-20 \mathrm{~km} \mathrm{~s}^{-1}$ to $40 \mathrm{~km} \mathrm{~s}^{-1}$, averaged over five consecutive channels $\left(\sim 5 \mathrm{~km} \mathrm{~s}^{-1}\right)$. The central LSR velocities are indicated above each panel. The grey-scale ranges between 40 and $300 \mathrm{~K} \mathrm{~km} \mathrm{~s}{ }^{-1}$. White contours are shown from $80 \mathrm{~K} \mathrm{~km} \mathrm{~s}^{-1}$ (except for the $2.1 \mathrm{~km} \mathrm{~s}^{-1}$ map, which starts at $100 \mathrm{~K} \mathrm{~km} \mathrm{~s}^{-1}$ ) and increases in steps of $40 \mathrm{~K} \mathrm{~km} \mathrm{~s}{ }^{-1}$. The $\lambda 6 \mathrm{~cm}$ total intensities smoothed to $18^{\prime}$ are superimposed as contours in black. The northern shell is located at about $67^{\circ}$ longitude and the southern shell at about $64^{\circ}$.

\subsection{Analysis of HI-observations}

Kalberla et al. (2005) published the Leiden/Argentina/Bonn (LAB) $\lambda 21 \mathrm{~cm}$ survey of neutral hydrogen, which covers the velocity range from $-450 \mathrm{~km} \mathrm{~s}^{-1}$ to $+400 \mathrm{~km} \mathrm{~s}^{-1}$ with a velocity resolution of $1.3 \mathrm{~km} \mathrm{~s}^{-1}$. The HPBW of the survey was 0.6 , spectra were taken every 0.5 and the rms brightness-temperature noise was about $0.07-0.09 \mathrm{~K}$.

A fully sampled HI-survey was also carried out with the DRAO 26-m telescope by Higgs et al. (2005). We found no significant difference between these two surveys, except that the DRAO HI-survey does not cover the very northern part of the SNR. Our HI-mass calculation is thus based on the LAB survey.

We extracted the HI-data of the G65.2+5.7 region from the LAB survey data-cube in the velocity range between $-20 \mathrm{~km} \mathrm{~s}^{-1}$ and $40 \mathrm{~km} \mathrm{~s}^{-1}$. The $0.8 \mathrm{kpc}$ distance corresponds to a velocity range $0-10 \mathrm{~km} \mathrm{~s}^{-1}$ according to the Galactic rotation model given by Fich et al. (1989) of $R_{0}=8.5 \mathrm{kpc}$ and $\Theta_{0}=$ $220 \mathrm{~km} \mathrm{~s}^{-1}$. Figure 10 displays the HI distribution after averaging five consecutive channels $\left(\sim 5 \mathrm{~km} \mathrm{~s}^{-1}\right)$ within the velocity interval from -20 to $40 \mathrm{~km} \mathrm{~s}^{-1}$. The central velocity of each image is indicated at the top. Contours of $\lambda 6 \mathrm{~cm}$ total intensity (smoothed to $18^{\prime}$ ) are superimposed to indicate the region of G65.2+5.7.

Although HI-fluctuations are visible across the entire field, we note signs of possible SNR interaction with the HI-gas in the images centered on $v=-3.1 \mathrm{~km} \mathrm{~s}^{-1}, 2.1 \mathrm{~km} \mathrm{~s}^{-1}$, and $v=$ $7.2 \mathrm{~km} \mathrm{~s}^{-1}$. The northern shell correlates with the lower velocity maps, while the southern shell correlates with the $7.2 \mathrm{~km} \mathrm{~s}^{-1}$ cloud. This may indicate that the northern shell is on the near side, or expands towards us, while the southern shell is on the far side or expands away from us.

In Fig. 11, we present the result for the integrated HI column densities over the velocity range -5 to $10 \mathrm{~km} \mathrm{~s}^{-1}$. We integrated HI column densities over the velocity range -5 to $10 \mathrm{~km} \mathrm{~s}^{-1}$, and subtracted the large-scale diffuse emission using the "unsharpmasking" procedure described by Sofue \& Reich (1979). A weak $\mathrm{HI}$ shell with an angular diameter of about $4^{\circ}$ is visible at the outer periphery of the radio continuum shell of SNR G65.2+5.7.

If a physical association exists, we would calculate the column density for the shell to be $2 \times 10^{20} \mathrm{~cm}^{-2}$. Assuming a thickness of the shell of $20 \%$ for a $2^{\circ}$ radius, this corresponds to a swept-up mass of about $1400 M_{\odot}$. The average density in the HI-shell is then about $1.2 \mathrm{~cm}^{-3}$. If this mass of neutral gas was originally uniformly distributed within a sphere of radius $2^{\circ}$, the 


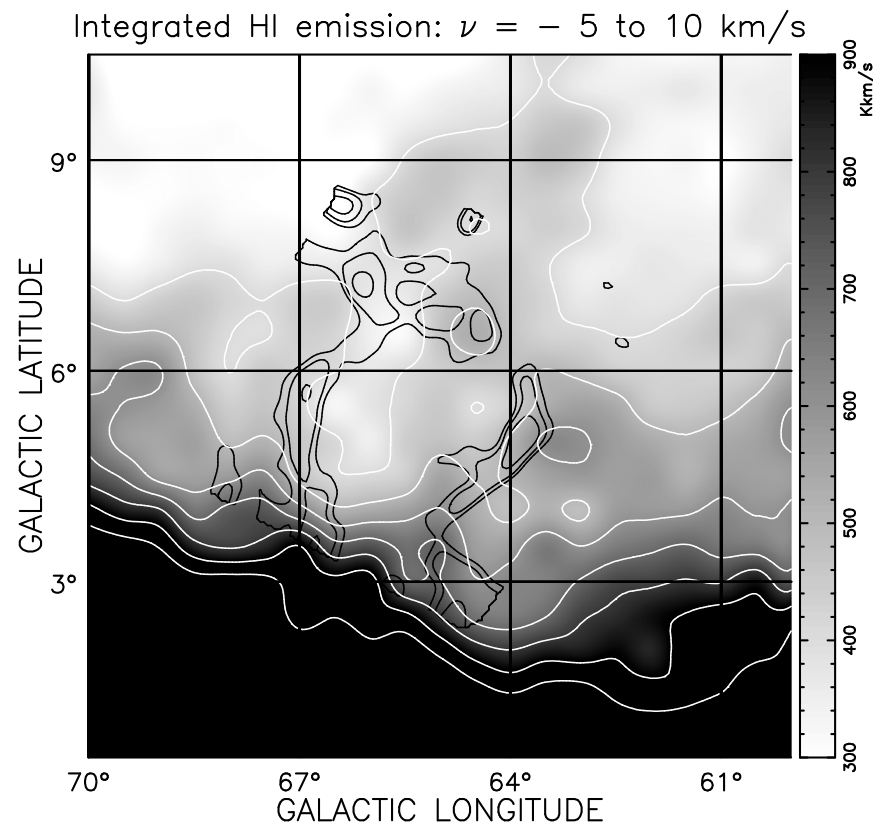

Fig. 11. Integrated HI-intensities for the velocity range from $-5 \mathrm{~km} \mathrm{~s}^{-1}$ to $10 \mathrm{~km} \mathrm{~s}^{-1}$ superimposed on with $\lambda 6 \mathrm{~cm}$ contours smoothed to $18^{\prime}$. White contours for HI intensities are shown from $400 \mathrm{~K} \mathrm{~km} \mathrm{~s}^{-1}$ and run in steps of $100 \mathrm{~K} \mathrm{~km} \mathrm{~s}^{-1}$.

pre-explosion ambient density would have been $0.6 \mathrm{~cm}^{-3}$, which is comparable to the value of $0.4 \mathrm{~cm}^{-3}$ estimated by Mason et al. (1979) for the X-ray emitting region. These densities are reasonable for a distance of $\sim 80$ pc away from the Galactic plane.

\subsection{Comparison with observations from other bands}

We show contours of $\lambda 11 \mathrm{~cm}$ total intensities of G65.2+5.7 superposed on an [O III] emission image by Boumis et al. (2004) in Fig. 12. The radio filaments are highly correlated with the [O III] filaments, which trace the fast SNR shock, except for the northwestern more diffuse part of the SNR. In this area, the SNR shock front seems to be distorted resulting in a less compressed magnetic field and a reduced efficiency in particle acceleration. We note that in some regions enhanced radio filamentary emission is seen without any corresponding optical emission. The magnetic fields might be stronger and/or the temperatures/densities lower in these bright radio filaments. This seems to be consistent with the measured incomplete cooling regions of G65.2+5.7 (Mavromatakis et al. 2002), which means that at least parts of the SNR are still in an adiabatic phase.

The G65.2+5.7 image (0.14-0.284 keV) from the ROSAT soft X-ray all-sky survey (Snowden et al. 1997) is shown in Fig. 13 (left panel) with contours of $\lambda 11 \mathrm{~cm}$ total intensity superimposed. The radio contours trace the outer SNR shock and clearly define an outer boundary of the X-ray emission. With its centrally filled X-ray thermal emission, G65.2+5.7 has been classified as a "thermal composite" SNR. Shelton et al. (2004) reviewed the evolution of "thermal composite" SNRs: A dense outer shell develops when the SNR enters the cooling phase, while the strong centrally peaked thermal X-ray emission may be explained by thermal conduction behind radiative shocks (Cox et al. 1999) and/or by the evaporation of interstellar clumps (White \& Long 1991). More studies are needed to develop a detailed scenario of the evolution.

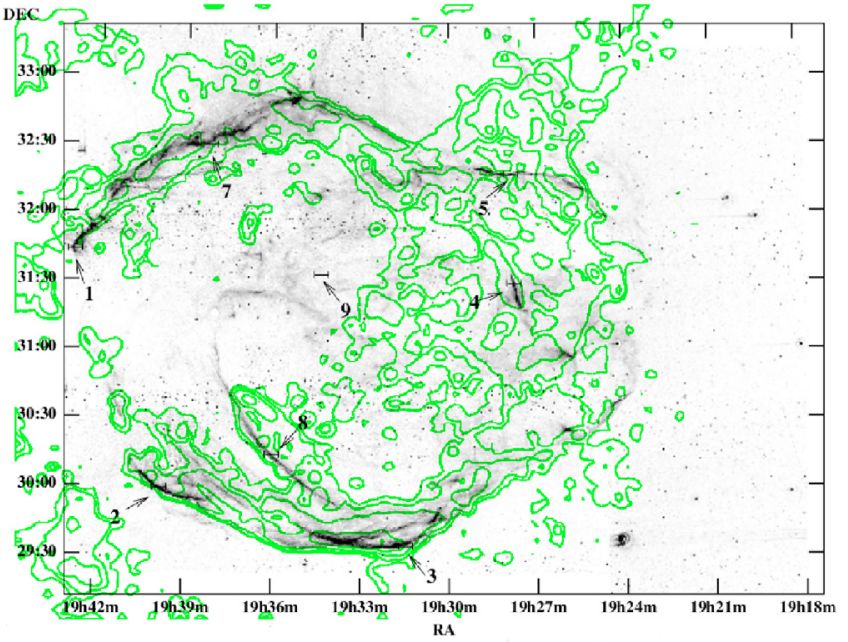

Fig. 12. Superposition of an [O III] image (Boumis et al. 2004) with $\lambda 11 \mathrm{~cm}$ total intensity contours. The $\lambda 11 \mathrm{~cm}$ map has the same levels as shown in Fig. 2 but the compact sources were subtracted.

We show $\lambda 11 \mathrm{~cm}$ contours superposed on an $\mathrm{H}_{\alpha}$ image of G65.2+5.7 (Finkbeiner 2003) in Fig. 13 (right panel). A good correlation exists between the radio structures and enhanced $\mathrm{H}_{\alpha}$ emission, in particular along the southern shell. We estimate the thermal electron density within the southern shell using the observed emission measure (EM); this is defined as the integral of the square of electron density of the source along the line-ofsight $l, \mathrm{EM}=n_{\mathrm{e}}^{2} l$, and related to the $\mathrm{H}_{\alpha}$ intensity by

$\mathrm{EM}=2.75 T_{4}^{0.9} I_{\mathrm{H} \alpha} \exp (\tau)$,

where $\mathrm{EM}$ is in units of $\mathrm{pc} \mathrm{cm}^{-6}, I_{\mathrm{H} \alpha}$ is the $\mathrm{H} \alpha$ intensity in Rayleigh, and the optical depth $\tau$ could be written in units of the product of $E(B-V)$ magnitudes of reddening and 2.44 according to Finkbeiner (2003).

Assuming an electron temperature of $10000 \mathrm{~K}$ for the thermal gas and given an $\mathrm{H}_{\alpha}$ intensity of 18 Rayleigh for the southern filament by filtering out the diffuse emission, we obtained an EM of about $145 \mathrm{pc} \mathrm{cm}^{-6}$ for a reddening $E(B-V)$ of 0.44 (Schlegel et al. 1998). We then obtained an electron density $n_{\mathrm{e}}$ of between $38 \mathrm{~cm}^{-3}$ and $22 \mathrm{~cm}^{-3}$ for a thickness of between $0.1 \mathrm{pc}$ and $0.3 \mathrm{pc}$. For the equipartition magnetic fields of $20 \mu \mathrm{G}$ to $50 \mu \mathrm{G}$ (filling factor 0.1 ), we may then calculate RMs ranging between $70 \mathrm{rad} \mathrm{m}^{-2}$ and $245 \mathrm{rad} \mathrm{m}^{-2}$ for the range of filament thicknesses and magnetic fields. We recall that for the case of a tangential magnetic field, the $\lambda 6 \mathrm{~cm}$ polarization angles constraint is about $\mathrm{RM} \leq 70 \mathrm{rad} \mathrm{m}^{-2}$.

We also investigated the distribution of CO-emission from the "Composite CO-Survey" (Dame et al. 1987) for the SNR region, but found no evidence of any associated emission.

\section{Summary}

We have presented high sensitivity maps of the SNR G65.2+5.7 for total and polarized intensities at $\lambda 6 \mathrm{~cm}$ and $\lambda 11 \mathrm{~cm}$. The spectral index of the integrated flux densities was found to be $\alpha=-0.58 \pm 0.07$, which is consistent with the value obtained by Reich et al. (1979). The distribution of spectral indices shows some variation, although within the error margins of the spectral index obtained for the integrated emission.

Strong polarized emission is observed from the southern filament of G65.2+5.7, where a high percentage polarization 

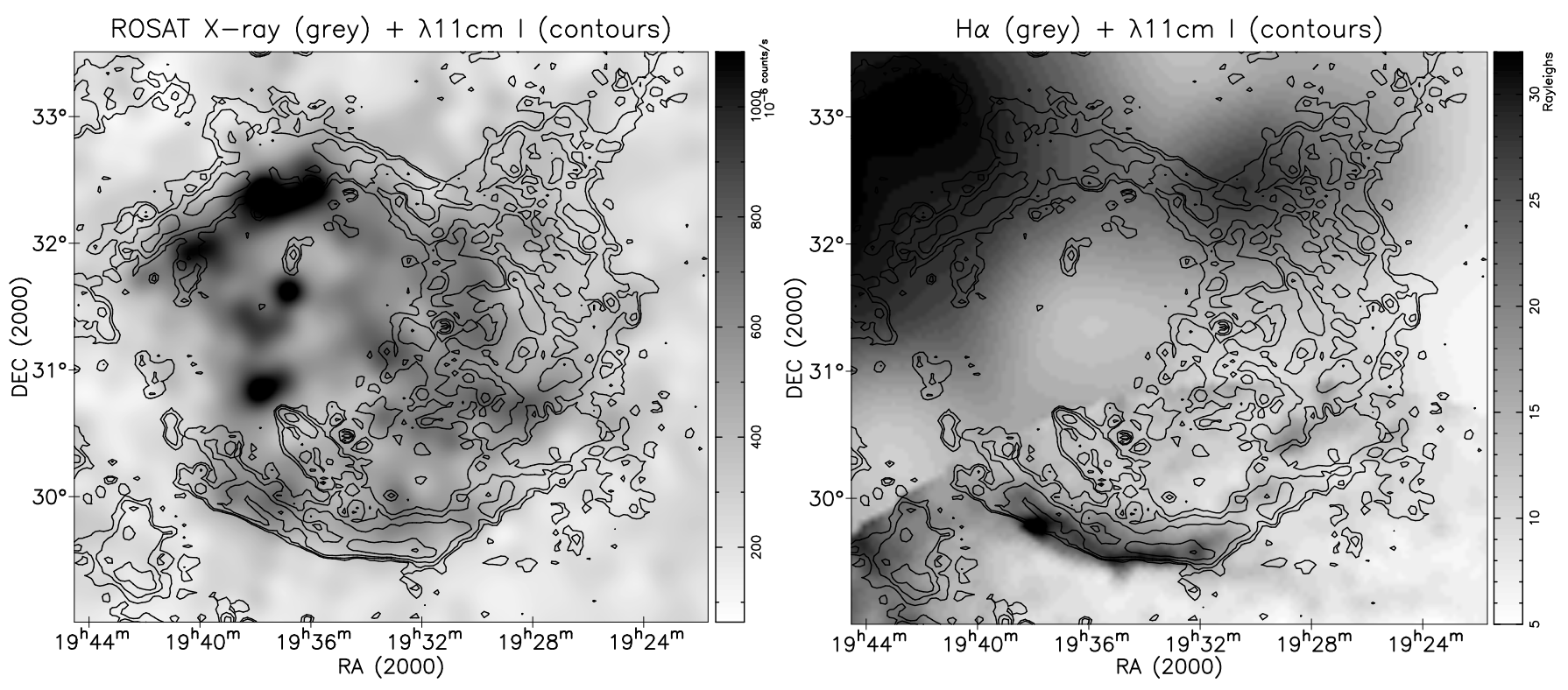

Fig. 13. Superimposed images of SNR G65.2+5.7. Left: ROSAT X-ray emission (Snowden et al. 1997) (grey-scale) smoothed to 10 arcmin angular resolution overlaid with $\lambda 11 \mathrm{~cm}$ total intensity contours. Right: $\mathrm{H}_{\alpha}$ map taken from Finkbeiner (2003) (grey-scale) overlaid with $\lambda 11 \mathrm{~cm}$ contours. Contour levels are as in Fig. 2. Note that compact sources were removed from the $\lambda 11 \mathrm{~cm}$ data shown here.

around $50 \%$ at $\lambda 6 \mathrm{~cm}$ indicates the presence of a strong regular magnetic field component. Clear depolarization along its outer rim is seen at $\lambda 11 \mathrm{~cm}$. The polarized emission at $\lambda 11 \mathrm{~cm}$ and $\lambda 6 \mathrm{~cm}$ are affected by a different amount of depolarization as well as confusion from diffuse Galactic polarized emission.

G65.2+5.7 is a faint large-diameter shell-type SNR with an exceptional low surface brightness in the radio range (Reich et al. 1979), but otherwise no unusual properties, e.g. no spectral bend as SNR S147 (Xiao et al. 2008). Except for possibly a few areas, G65.2+5.7 has almost entered the cooling phase. The strong depolarization along the outer SNR shell seems to support the classification of G65.2+5.7 as a rare example of a "thermal composite" SNR based on X-ray data.

Acknowledgements. We thank Dr. XiaoHui Sun for useful discussions and Dr. Patricia Reich for reading and comments on the manuscript. We are very grateful to the referee Dr. Tyler Foster for constructive comments, which clearly improved the manuscript. The $\lambda 6 \mathrm{~cm}$ data were obtained with a receiver system from the MPIfR mounted at the Nanshan $25 \mathrm{~m}$ telesope at the Urumqi Observatory of NAOC. The nice receiver was constructed by Mr. Otmar Lochner of MPIfR, and well-maintained by Mr. M. Z. Chen and J. Ma of Urumqi observatory. The $\lambda 11 \mathrm{~cm}$ observations are based on observations with the $100-\mathrm{m}$ telescope of the MPIfR (Max-Planck-Institut für Radioastronomie) at Effelsberg. This research work was supported by the National Natural Science Foundation of China (10773016, 10833003 and 10821061), and the Partner group of the MPIfR at NAOC in the frame of the exchange program between MPG and CAS for a number of bilateral visits.

\section{References}

Boumis, P., Meaburn, J., Lopez, J. A., et al. 2004, A\&A, 424, 583 Camilo, F., Nice, D. J., Shrauner, J. A., \& Taylor, J. H. 1996, ApJ, 469, 819 Condon, J. J., Cotton, W. D., Greisen, E. W., et al. 1998, AJ, 115, 1693 Cordes, J. M., \& Lazio, T. J. W. 2002, preprint [arXiv: astro-ph/0207156] Cox, D. P., Shelton, R. L., Maciejewski, W., et al. 1999, ApJ, 524, 179 Dame, T. M., Ungerechts, H., Cohen, R. S., et al. 1987, ApJ, 322, 706 Emerson, D. T., \& Gräve, R. 1988, A\&A, 190, 353

Fich, M., Blitz, L., \& Stark, A. A. 1989, ApJ, 342, 272

Finkbeiner, D. P. 2003, ApJS, 146, 407
Foster, T. 2005, A\&A, 441, 1043

Fürst, E., \& Reich, W. 2004, in The Magnetized Interstellar Medium, ed. B. Uyanıker, W. Reich, \& R. Wielebinski, Copernicus GmbH, 141

Gull, T. R., Kirshner, R. P., \& Parker, R. A. R. 1977, ApJ, 215, L69

Han, J. L., Manchester, R. N., Lyne, A. G., Qiao, G. J., \& van Straten, W. 2006, ApJ, 642, 868

Haslam, C. G. T., Wilson W. E., Graham, D. A., \& Hunt, G. C. 1974, A\&AS, 13, 359

Higgs, L. A., Landecker, T. L., Asgekar, A., et al. 2005, AJ, 129, 2750

Kalberla, P. M. W., Burton, W. B., Hartmann, D., et al. 2005, A\&A, 440, 775

Kovalenko, A. V., Pynzaŕ, A. V., \& Udal'Tsov, V. A. 1994, AZh, 71, 110

Mason, K. O., Kahn, S. M., Charles, P. A., \& Lampton, M. L. 1979, ApJ, 230, 163

Mavromatakis, F., Boumis, P., Papamastorakis, J., \& Ventura, J. 2002, A\&A, 388,355

Orlando, S., Bocchino, F., Reale, F., Peres, G., \& Petruk, O. 2007, A\&A, 470, 927

Page, L., Hinshaw, G., Komatsu, E., et al. 2007, ApJS, 170, 335

Reich, P., \& Reich, W. 1988, A\&AS, 74, 7

Reich, W. 2006, in Cosmic Polarization, ed. R. Fabbri, Research Signpost, 91 [arXiv: astro-ph/0603465]

Reich, W., Berkhuijsen, E. M., \& Sofue, Y. 1979, A\&A, 72, 270

Reich, W., Reich, P., \& Fürst, E. 1990, A\&AS, 83, 539

Reich, W., Fürst, E., \& Arnal, E. M. 1992, A\&A, 256, 214

Reich, W., Fürst, E., Reich, P., et al. 2004, in The Magnetized Interstellar Medium, ed. B. Uyanıker, W. Reich, \& R. Wielebinski, Copernicus GmbH, 57

Schaudel, D., Becker, W., Lu, F., \& Aschenbach, B. 2002, 34th COSPAR Scientific Assembly, Houston/USA, meeting abstract

Schlegel, D., Finkbeiner, D. P., \& Davis, M. 1998, ApJ, 500, 525

Shelton, R. L., Kuntz, K. D., \& Petre, R. 2004, ApJ, 615, 275

Snowden, S. L., Egger, R., Freyberg, M. J., et al. 1997, ApJ, 485, 125

Sofue, Y., \& Reich, W. 1979, A\&AS, 38, 251

Sun, X. H., Reich, W., Han, J. L., Reich, P., \& Wielebinski, R. 2006, A\&A, 447, 947

Sun, X. H., Han, J. L., Reich, W., et al. 2007, A\&A, 463, 993

Turtle, A. Y., Pugh, G. F., Kenderdine, S., \& Pauliny-Toth, I. I. K. 1962, MNRAS, 124, 297

White, R. L., \& Long, K. S. 1991, ApJ, 373, 543

Wolleben, M., Landecker, T. L., Reich, W., \& Wielebinski, R. 2006, A\&A, 448, 411

Xiao, L., Fürst, E., Reich, W., \& Han, J. L. 2008, A\&A, 482, 783

Xu, J. W., Han, J. L., Sun, X. H., et al. 2007, A\&A, 470, 969 